\title{
Changes in the Gap Junction of Rat Exocrine Pancreatic Cells Induced by Long-term Ethanol Ingestion
}

\author{
Toru Sato \\ Shizugawa Public General Hospital, Shizugawa, Miyagi \\ 986-07
}

\begin{abstract}
Sato, T. Changes in the Gap Junction of Rat Exocrine Pancreatic Cells Induced by Long-term Ethanol Ingestion. Tohoku J. Exp. Med., 1994, 172 (1), 3949 - Fourteen Male Wistar rats were orally administered $20 \%$ ethanol ad libitum in place of water. After 1,2,3,6 and 12 months of ethanol ingestion, the gap junctions of pancreatic acinar and ductal cells were observed by transparent electron microscopy. The result was compared with fifteen control animals. In the control, the ductal cells proved to have only small sized (0.1 micron in length) gap junction located in the apical domain as a part of junctional complex. While, in the acinar cells, not only small but large-sized gap junctions several microns in length were observed in the baso-lateral domain. After 3 months of ethanol ingestion, the gap of these two types of gap junctions was replaced with a continuous, granular structure. In addition, irregularly running gap junctions were observed after 6 months of ethanol ingestion. The functional implication of these morphological changes is not clear. _— ethanol; alcoholic pancreatitis; electron microscopy; gap junction; intercellular junction
\end{abstract}

In a previous paper dealing with the changes of rat exocrine pancreatic cells induced by long-term ingestion of ethanol (Nakamura et al. 1990), it was reported that 1) the tight junction was confirmable only between ductular cells, including centroacinar cells, but not between acinar cells, 2) after 1 to 2 months of ethanol ingestion, tight junctions increased in number with hypertrophy of the interdigitation and, 3) after 3 months, this structure rapidly decreased in number with atrophy of the interdigitation.

The present paper describes, as an extension of the above study, the changes of gap junction of the pancreatic exocrine cells with ethanol ingestion. The paper reports at the same time that in the acinar cells of control rats there are two kinds of gap junctions, the first of which was located in the baso-lateral domain only of acinar cells and was extending over an extraordinary length (several microns in length in ultrathin section). The second was located in the apical part of acinar as well as ductal cells and was confined to a small range (about 0.1 micron in

Received May 25, 1993; revision accepted for publication December 20, 1993.

Reprint requests: Toru Sato, M.D., The Third Department of Medicine, Akita University School of Medicine, 1-1-1 Hondo, Akita 010, Japan. 
length). After 3 months of ethanol ingestion, these gap junctions were shown undergoing a peculiar structural change where the normally visible gap was replaced with a coarsely granular appearance.

\section{Materials and Methods}

A detailed description of the materials and methods used was given previously (Kakizaki et al. 1987; Nakamura et al. 1990). Fourteen male Wistar rats were fed with a standard diet and water provided ad libitum for 4 months, after which the water was replaced with

TABLE 1. Number of rats in dach breeding period

\begin{tabular}{lllllll}
\hline & \multicolumn{5}{c}{ Breeding period for all the animals } \\
& 5 & 6 & 7 & 10 & 16 & \multirow{2}{*}{ Total } \\
\cline { 2 - 5 } & \multicolumn{4}{c}{ Period of ethanol intake for the ethanol group } & \\
& 1 & 2 & 3 & 6 & 12 & \\
\hline Control group & 3 & 3 & 3 & 3 & 3 & 15 \\
Ethanol group & 3 & 2 & 3 & 3 & 3 & 14 \\
\hline
\end{tabular}

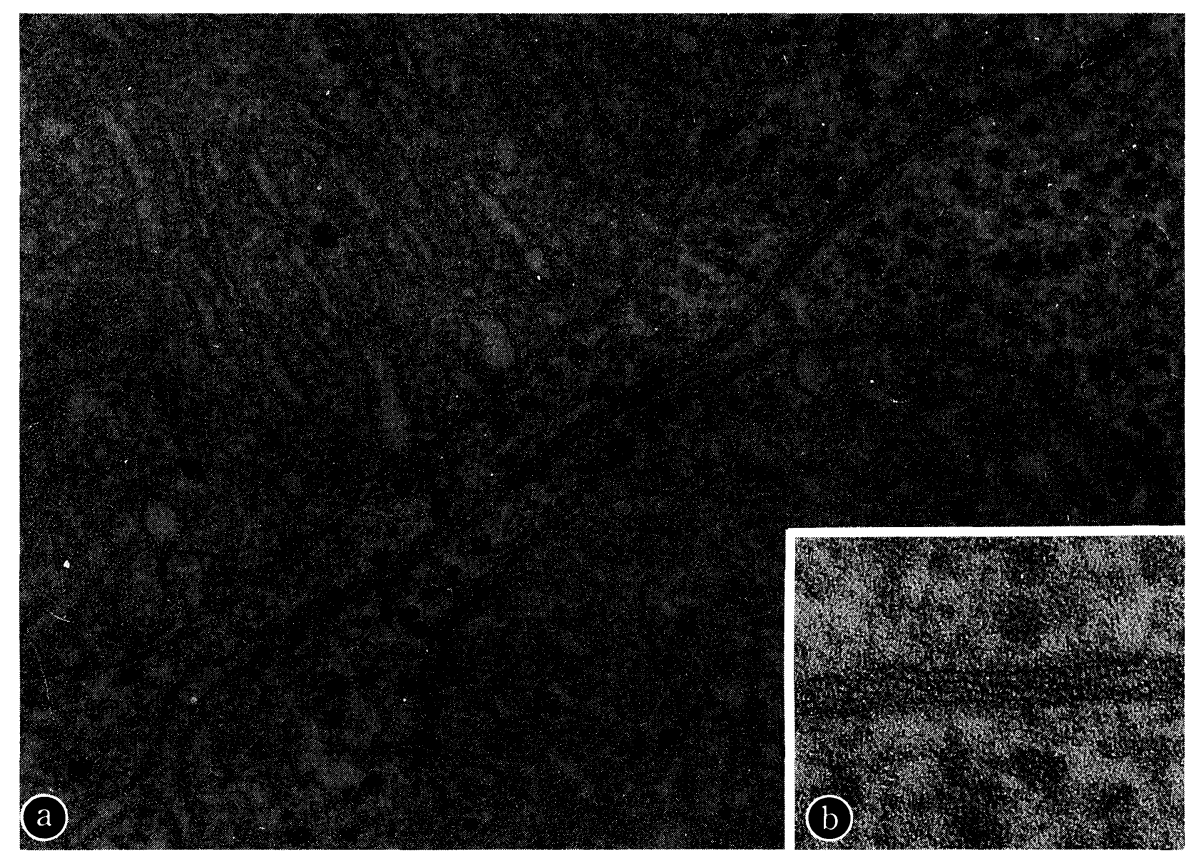

Fig. 1. a: Acinar cells of a control rat. Between mitochondria, a large-sized gap junction is observed. $\times 100,000$. b: (Inlet) Higher magnification of part of a. The gap structure is observed distinctly. $\times 300,000$. 
$20 \%$ ethanol. Animals were studied after 1, 2, 3, 6 and 12 months of ethanol ingestion $(n=$ 3 for all groups except the 2-months group, where $n=2$ ). Another series of fifteen control animals received a standard diet and water for 5, 6, 7, 10 and 16 months $(n=3$ for each group) (Table 1). Pancreatic specimens were obtained under ether anesthesia, fixed in a cacodylate-buffered mixture of $4 \%$ paraformaldehyde and $5 \%$ glutaraldehyde, then postfixed in cacodylate-buffered $1 \%$ osmium tetroxide and embedded in Epon. Ultra-thin sections were cut from three Epon blocks from each animal and stained with alcoholic uranyl acetate and lead citrate and observed with a JEM 100 S-X electron microscope (JEOL, Tokyo). Some photomicrographs were taken at oblique angles with the use of a simple apparatus for tilting the specimen in the electron microscope and some were taken from serial ultrathin sections. Photomicrographs were used for observation as well as measurement of the thickness of the intercellular junctions and other structures under a dissecting microscope equipped with micrometer, at magnifications of 10 to 20 . The thickness of the plasma membrane of the acinar cells of control rats, $7 \mathrm{~nm}$, was used as a standard.

\section{RESULTS}

In the control animals, two types of gap junctions were found to exist between exocrine pancreatic cells. They differed in their location and size. The first type was found in the baso-lateral domain of the acinar cells and was

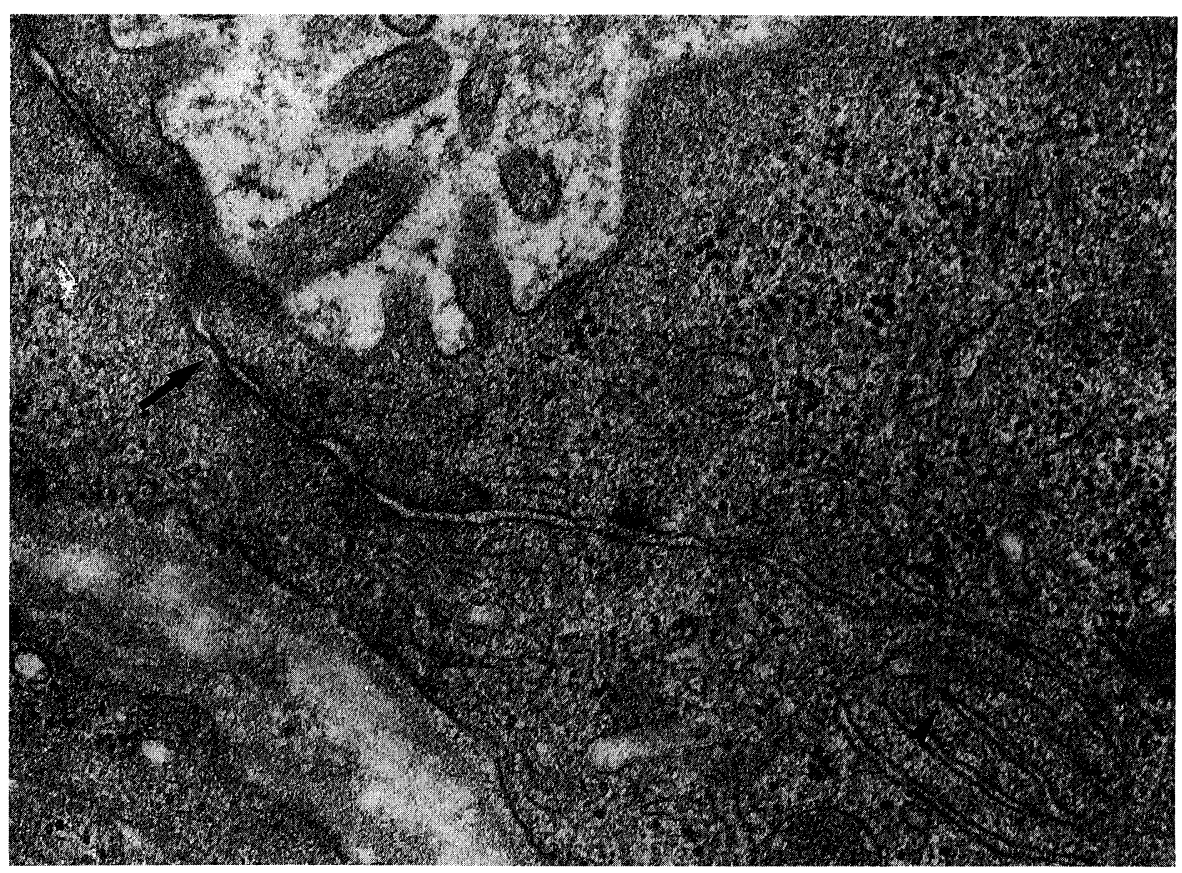

Fig. 2. Ductular cells of a control rat. In the apical region, a small-sized gap junction (arrow) is observed together with the occluding junction and the desmosome. No attachment of the filamentous structure to the gap junction is observed. A tight junction is formed between the baso-lateral interdigitations (arrowhead). No filamentous attachment is observed on the tight junction. $\times 40,000$. 
extending along several microns in a thin section. In places where the opposing cytoplasmic membranes were cross-sectioned, the gap structure was easily confirmable, leaving no difficulty in differentiating from other junctional structures (Fig. 1).

The second type was located in the apical portion of acinar as well as ductal cells (Fig. 2). This type of junction was usually observed to form a junctional complex together with an occluding junction and an adhering junction. This second type of gap junction was shown occupying only a small area within 0.1 micron in length. Electron micrographs from serial sections of ductal cells containing this type of junctional complex are given in Figs. 3a, b, c and d. Judging from these figures, this type of junction appears not to be a belt-like but a macular structure. When this structure was cut at an oblique angle in an ultra-thin section or when it was observed in a low magnification electron micrograph, the gap structure was hardly discernible. Even in such cases, the gap structure could be visualized by tilting the specimen in the electron microscope. Moreover, measurement of the distance between the opposing inner leaflets of the cell membanes revealed that it was constantly 16 to $18 \mathrm{~nm}$. This was clearly different from the distance of opposing inner leaflets of the occluding junction (20$22 \mathrm{~nm}$ ) or the tight junction (14-16 nm) reported by Nakumura et al. (1990). The anchoring cytoskeleton was, as the first type gap junction, completely absent in
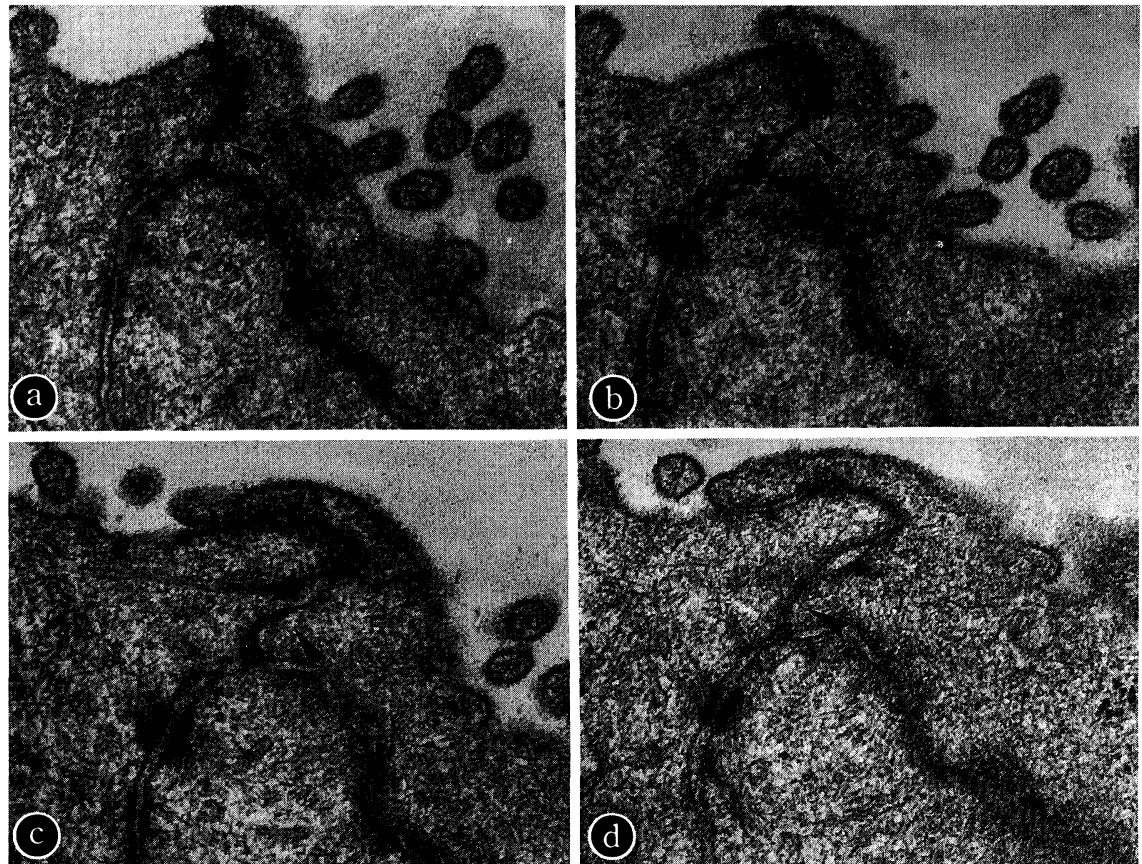

Fig. 3. a-d: Serial sections of the ductular cells of a control rat. From these, a gap junction is supposed to distribute as a small spot (arrowhead). $\quad \times 45,000$. 


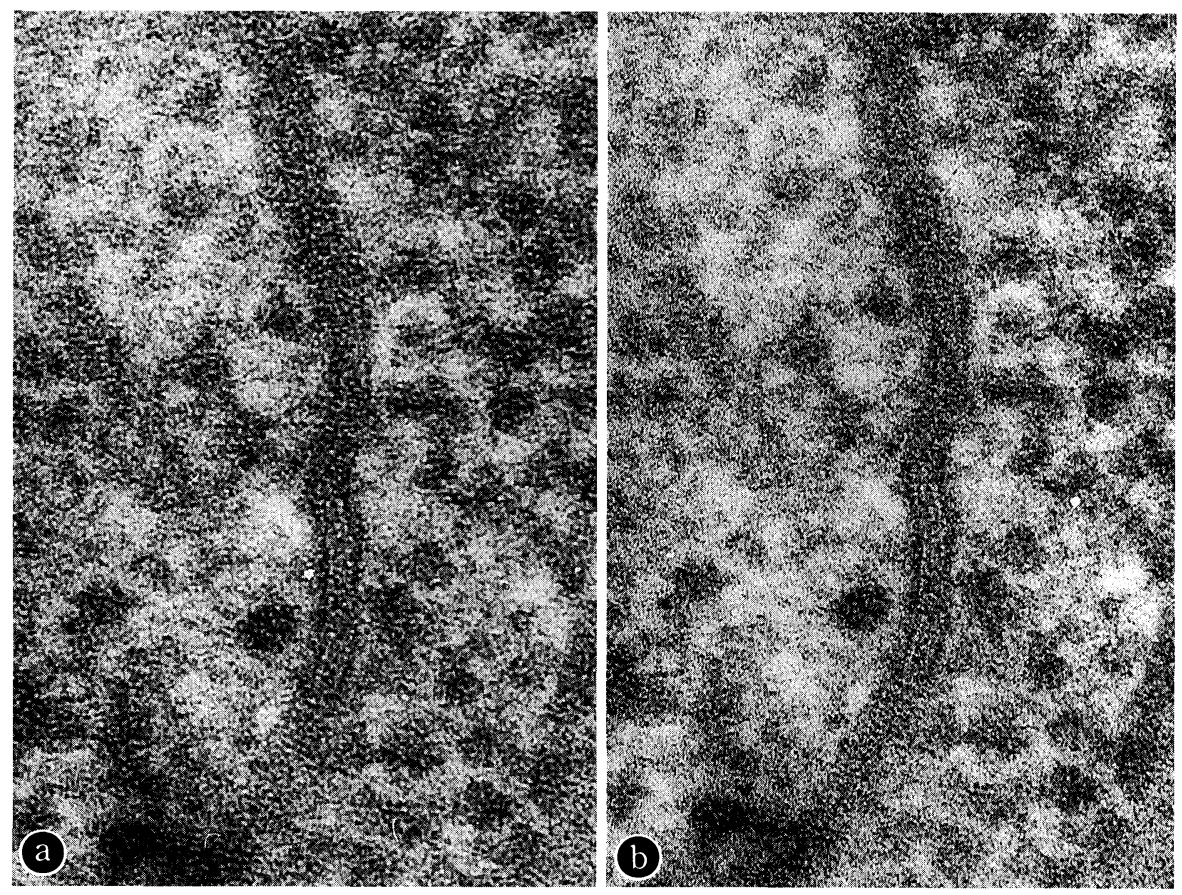

Fig. 4. Tilted figures of the large-sized gap junction of a control rat (tilt angle: $\left.\mathrm{a},-10^{\circ} ; \mathrm{b},+10^{\circ}\right)$. The gap structure does not show granular change. $\times 250,000$.

this second type too. Conversely, the occluding junction contained actomyosintype $7 \mathrm{~nm}$ microfilaments (Figs. 2, 3a, 3b, 3c and $3 \mathrm{~d}$ ).

After 3 months of ingestion, the gap of the above two kinds of gap junctions changed to a continuous, coarsely granulated appearance. This change was observed focally at the level of acinus but was distributed throughout the whole of the pancreas. The change in gap structure was not an artifact created by oblique sectioning; it was demonstrated by tilting the figures obtained from control (Fig. 4) and ethanol-fed animals (Fig. 5). In addition, varying the level of focus did not reveal basically different figures (Figs. 6a and b). In animals after 2 months of ethanol ingestion, a similar but partial change of the gap was observed (Fig. 7). After 6 months of ethanol ingestion, irregularly ramified gap junctions emerged, as shown by the irregularly running cell membranes (Fig. 8).

Even in the gap junctions revealing these morphological changes, the distance between a pair of opposing inner leaflets of cell membranes remained 16 to $18 \mathrm{~nm}$, the same as that of control animals.

\section{Discussion}

The terminology employed here and in the previous paper (Nakamura et al. 1990) for certain intercellular junctions does not always conform to the conven- 

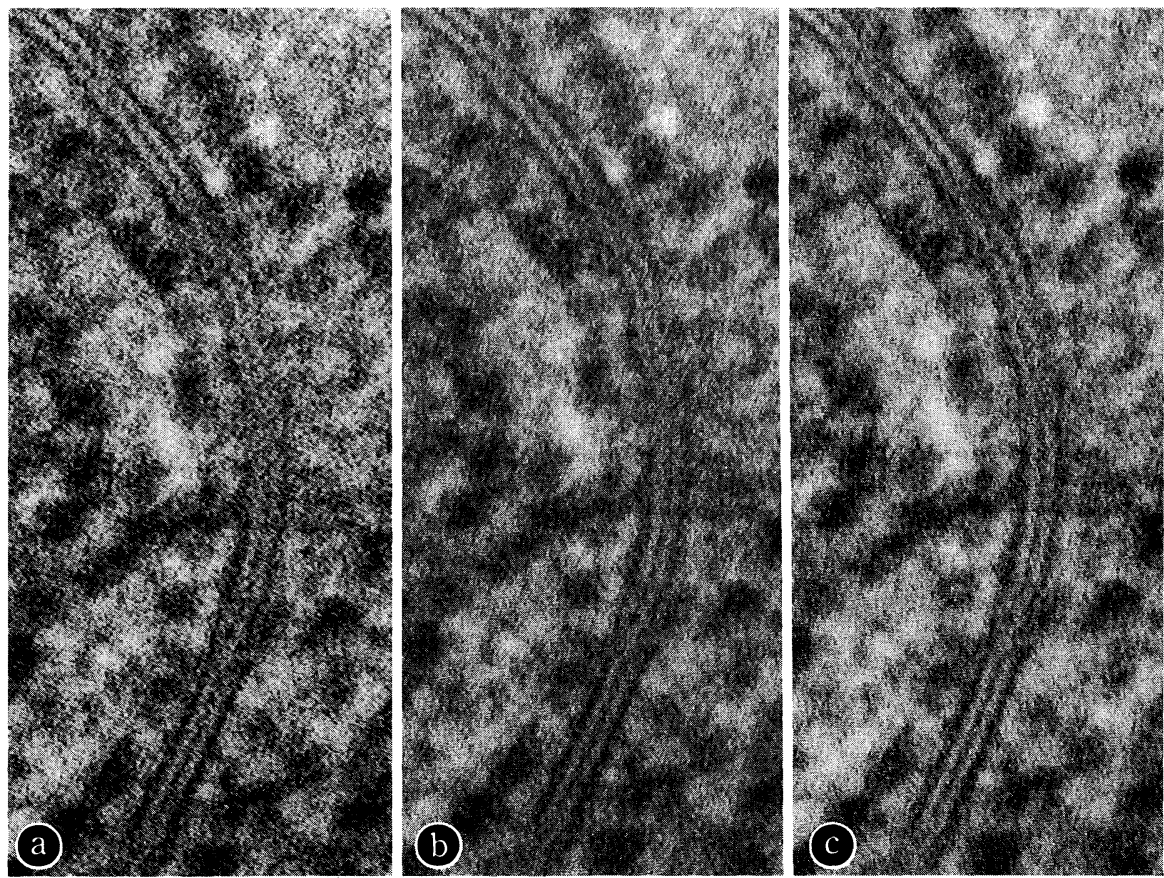

Fig. 5. Tilted figures of large-sized gap junction of rat administered ethanol for 3 months (tilting angle: $\mathrm{a},-10^{\circ} ; \mathrm{b}, 0^{\circ} ; \mathrm{c},+10^{\circ}$ ). Changing the tilting angles does not reveal a gap structure. $\times 250,000$.
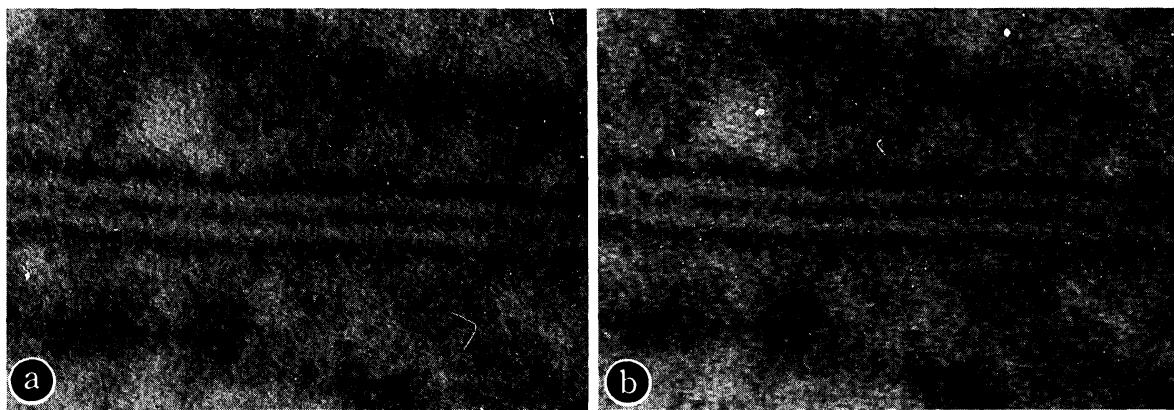

Fig. 6. a, b: Gap junction of the acinar cell of rat administered ethanol for 3 months. These figures were taken changing the level of focus of the electron microscope. The gap structure is not observed in either figure. $\times 500,000$.

tional usage, and therefore may require a small comment. The author thinks it pertinent to define the junctions which show punctate membrane "kisses" as follows: The "tight junction" is a junction with a width of 14 to $16 \mathrm{~nm}$ between the two opposing inner leaflets of the plasma menbranes at the point of the "kiss" and appears as a simple membrane fusion with no particular central substructure; the "gap junction" has a width of 16 to $18 \mathrm{~nm}$ with a 2 to $4 \mathrm{~nm}$ gap; the 


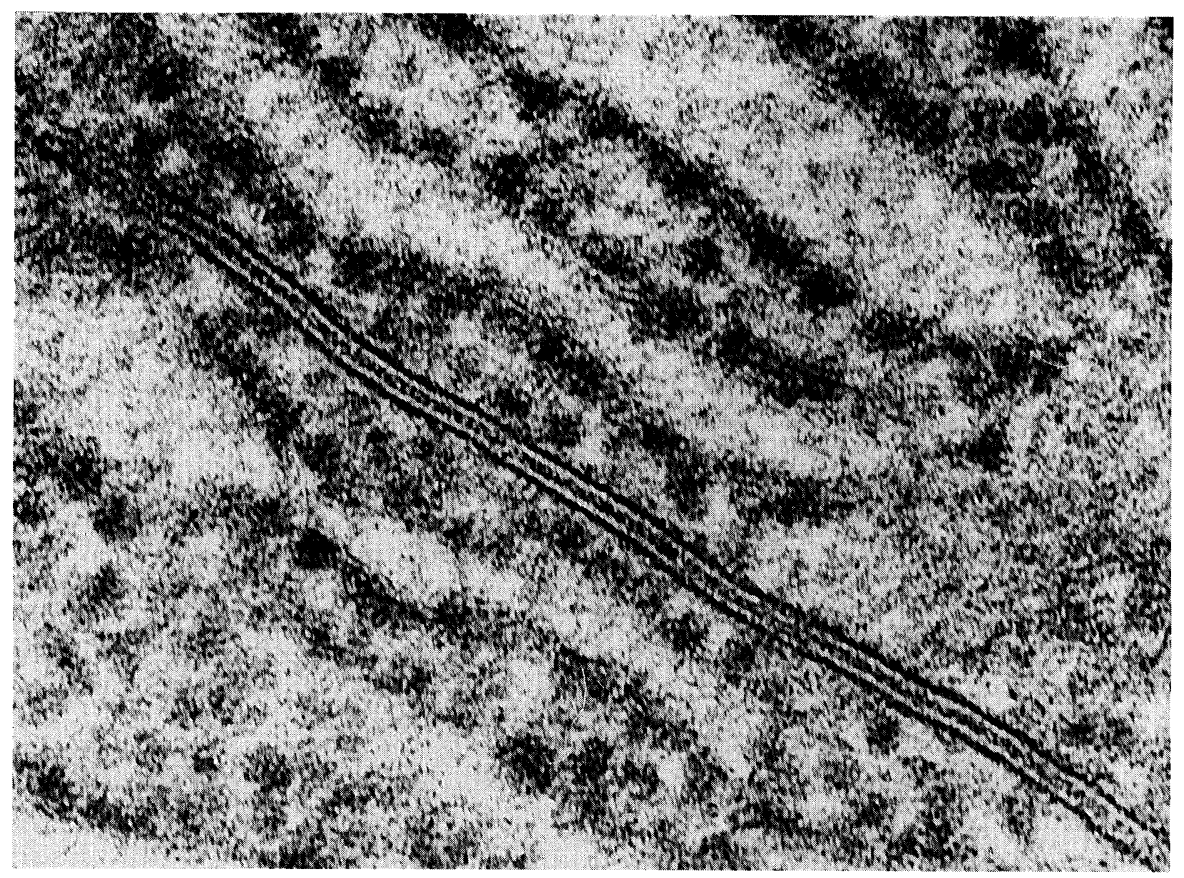

Fig. 7. The gap junction of rat acinar cells administered ethanol for 2 months. The gap structure becomes focally indistinct. $\times 250,000$.

"occluding junction" has a width of 20 to $22 \mathrm{~nm}$ with a 5 to $7 \mathrm{~nm}$ ovoid core. Interaction of the cytoskeleton at the cytoplasmic face is observed in the occluding junction but not in tight or gap junctions. Fig. 9 illustrates the differences among these junctions based on the above criteria. It should be noted here that the "tight" junctions in the author's sense are, as described previously by Nakamura et al. (1990), those only found in the interdigitated baso-lateral domain of the ductular cells.

Based on these criteria, i.e., on the width of the junction, difference in the central structure and cytoskeletal interaction, the author described in addition to the usual large gap junction the small one. The latter, with the junctional complex and being hardly recognizable as a gap junction in low magnification electron micrographs, may have been erroneously regarded as an occluding junction or its part. Stevenson and Goodenough (1984), in their electron micrographs of freeze-fractured liver cells, showed the assemblage of gap junction particles adjacent to the grooves or fibrils of zonula occludens (occluding junction). In their transparent electron microscopic pictures, however, they did not refer to the presence of gap junction in junctional complexes. Bruzzone and Meda (1988) reported that in pancreatic B-cells, gap junctions were usually smaller than those of other secretory epithelia. There has been no previous description of what we call the "small gap junction" of the acinar as well as the ductular cells. It is not 


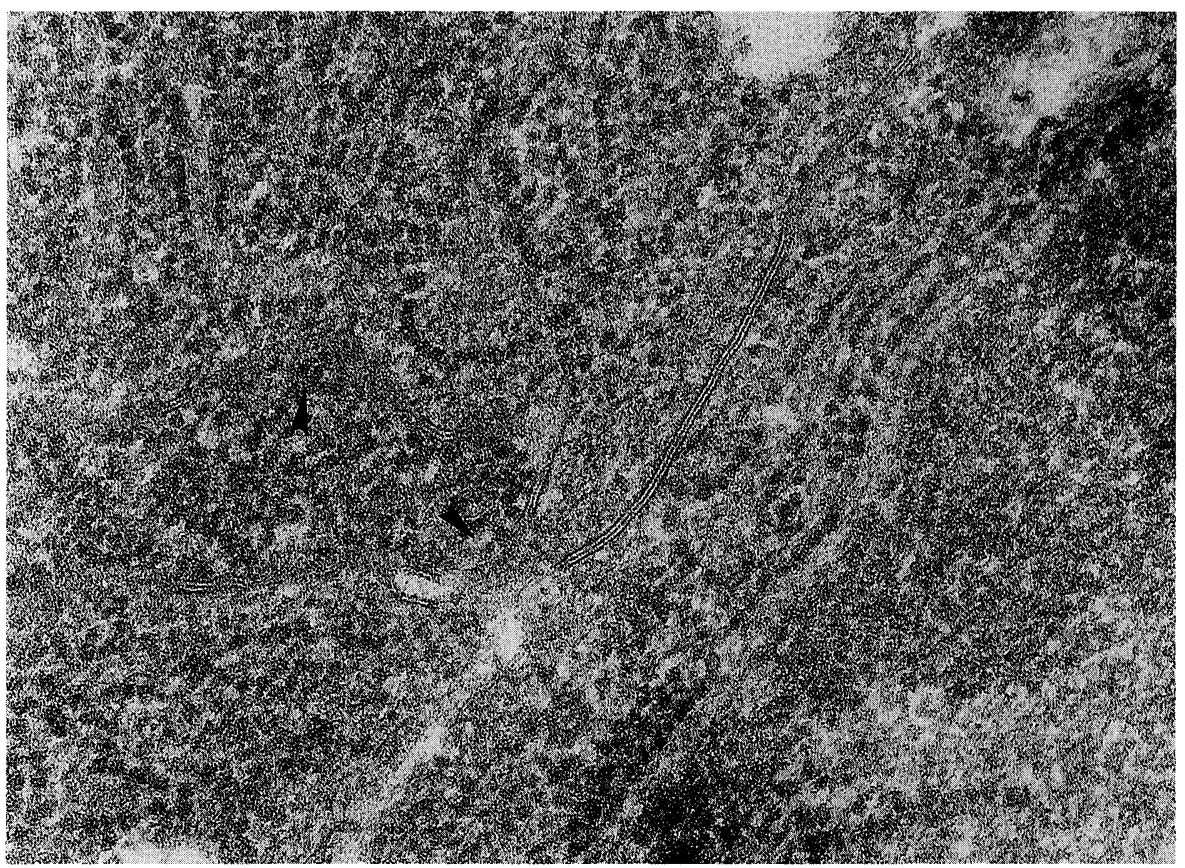

Fig. 8. Acinar cells of a rat administered ethanol for 6 months. The large-sized gap junction is observed runing irregularly (arrowheads). The gap structure is also unusual. $\times 80,000$.

clear whether there is difference if any in substructure and function between the small and the large gap junctions, the former being located in the apical domain of the acinar and ductular cells and the latter in the lateral area only of the acinar cells.

On the tight junction, that is the occluding junction according to the author's definition, a large number of biochemical, physiological and morphological studies have been published. As shown above, the tight junction (occluding junction) appears as a series of punctate membrane "kisses" where the extracellular space is eliminated (Figs. 10 and 11). Citi (1993) assumes that the tight junction (occluding junction) functions as a barrier preventing free diffusion of lipids in the exoplasmic membrane leaflets, which can cause intermixing of the specific apical and baso-lateral membrane proteins. A putative transmembrane tight junction protein may be located at the points of "kisses." This putative transmembrane protein particles may further interact with particulate elements such as ZO-1, ZO-2 and 7H-6 and filamentous elements as cingulin, actin, etc. Yet, these chemically identified tight junction-related substances have not been correlated with morphological findings. Also, whether the 5 to $7 \mathrm{~nm}$ core observed in the occluding junction (Fig. 1b) corresponds to the strands or grooves visualized by freeze-fracture technique, is not certain. 


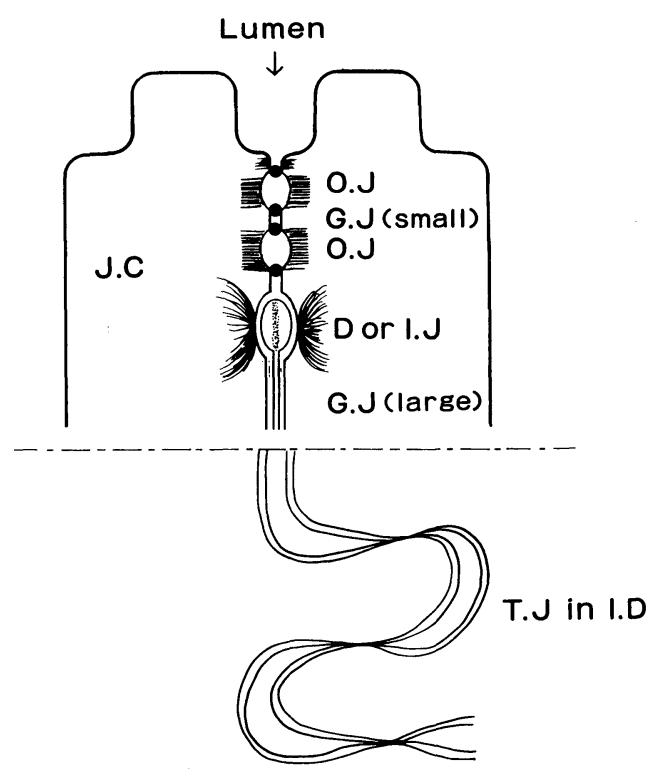

Fig. 9. A scheme illustrating various types of intercellular junctions. D, desmosome (10 nm intermediate filament); G.J., gap junction; I.J., intermediate junction ( $7 \mathrm{~nm}$ microfilament); I.D., interdigitation; J.C., junctional complex; O.J., occluding junction; T.J., tight junction.

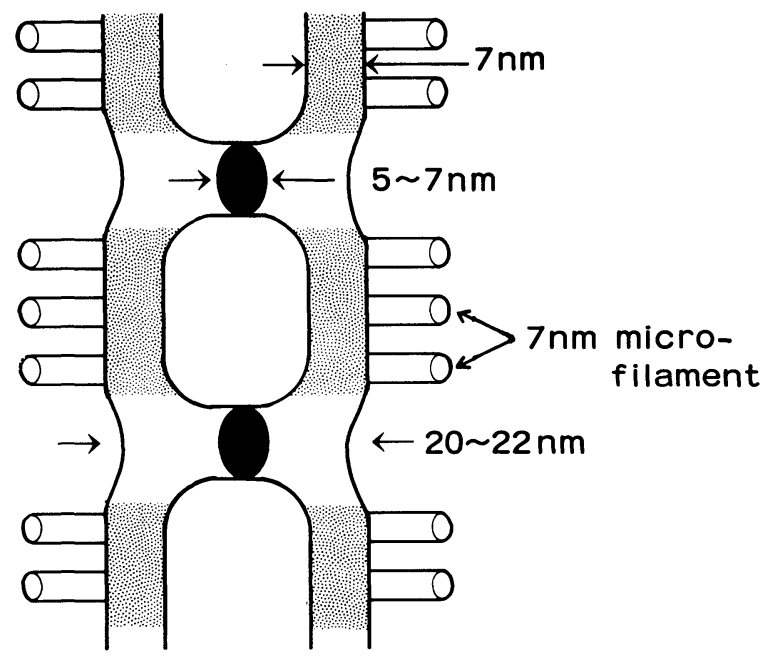

Fig. 10. Schematic illustrations of occluding junction. Plasma membranes probably correspond to the groove or strand observed in freeze fractured specimen. 
From a morphological viewpoint, the structure we described as "tight" junction (Nakamura et al. 1990) is a simple punctate membrane fusion which, in the pancreatic ductular cells, would make the intercellular space more complicated, like a labyrinth formed between interdigitated baso-lateral domains (Fig. 11a). This specialized membrane structure may be advantageous for exchanging electrolyte and fluid between pancreatic ductular cells. In fact, the interdigitation associated with the "tight" junction of the pancreatic ductular cells becomes hypertrophic as the number of "tight" junctions increases after 1 and 2 months of ethanol ingestion; over 3 months, the interdigitation becomes atrophic and the number of "tight" junctions markedly decreases (Nakamura et al. 1990). These also appear corresponding to the changes in the excretion of bicarbonate salts in pancreatic juice (Bordalo et al. 1976; Dreiling and Bordalo 1973).

After long-term ethanol ingestion, the gap junction of the pancreatic exocrine cells was shown to undergo a coarsely granular change of gap (Figs. 11b and c). The gap junction is discriminated from the "tight" junction in the pancreas firstly by the difference in its distribution. Secondly, even in the gap junction showing the granular change of gap induced by ethanol, the width of the junction remains
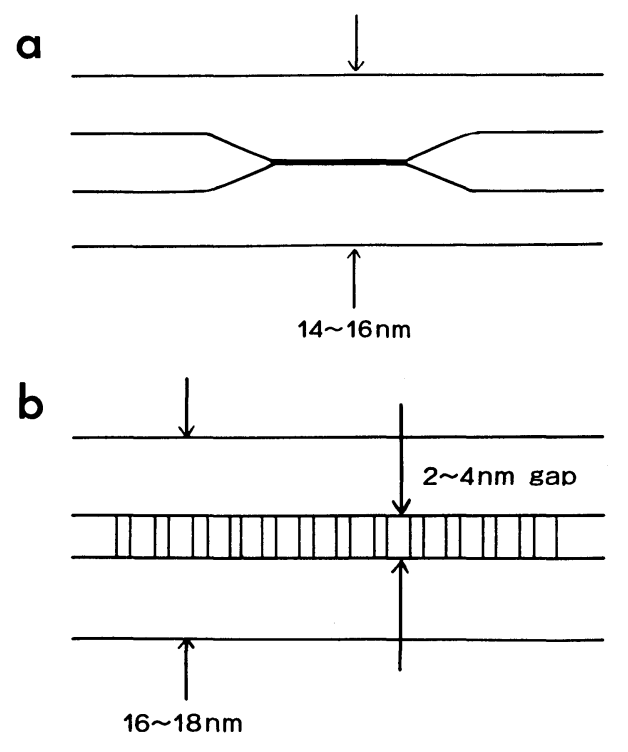

C

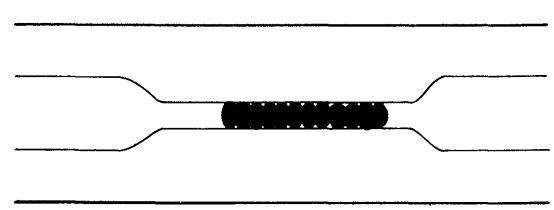

Fig. 11. Schematic illustrations of a, tight junction; b, gap junction; c, gap junction induced by ethanol ingestion. 
constantly 16 to $18 \mathrm{~nm}$ (Fig. 2). Granularly changed gap structures were further compared with normal ones by tilting the specimens or changing the microscope focus. However, no fundamental characteristics of gap junction proved to change.

Peracchia (1991), demonstrating that anesthetics such as heptanol and halothane block the gap junction channels of crayfish axons, assumed this to be an evidence for the presence of direct interaction between anesthetics and amphiphilic chains of gap junction proteins. It can be possible that the granular change of gap induced by alcohol has a similar functional implication. With regard to this, the present study poses another problem: how, in heavily alcoholintoxicated individuals, anesthetics are transmitted through the gap junction. Also, how a gap junction, with the gap changed into a coarsely granular structure, can appear in freeze-fractured specimens, remains for future studies.

\section{Acknowledgments}

I express my gratitude to Professor Akira Miura, Department of Internal Medicine, Akita University School of Medicine, Akita, for critical review of the manuscript, and wish to thank Dr. Katsuhiro Nakamura, Department of Pathology, Sendai Shakaihoken Hospital, Sendai and Dr. Goro Kakizaki, Shizugawa Public General Hospital, Shizugawa, for their help.

\section{References}

1) Bordalo, O., Noronha, M., Lamy, J. \& Dreiling, D.A. (1976) Standard and augmented secretin testing in chronic pancreatic alcoholic disease. Am. J. Gasroenterol., 64, 125-132.

2) Bruzzone, R. \& Meda, P. (1988) The gap junction: A channel for multiple functions? Eur. J. Clin. Invest., 18, 444-453.

3) Dreiling, D.A. \& Bordaro, O. (1973) Secretory patterns in minimal pancreatic inflammatory pathologies. Am. J. Gastroenterol., 60, 60-69.

4) Kakizaki, G., Sasahara, M., Aikawa T., Matsuo, M., Sugawara, Y., Nakamura, K., Endo, S. \& Ito, Y. (1987) On the pathogenesis of chronic alcoholic pancreatitis from the viewpoint of experimental results in rats. Int. J. Pancreatol., 2, 101-116.

5) Nakamura, K., Sato, T. \& Kakizaki, G. (1990) Changes in the intercellular junction of rat exocrine pancreatic cells induced by long term ethanol ingestion. Tohoku $J$. Exp. Med., 161, 283-301.

6) Peracchia, C. (1991) Effects of the anesthetics heptanol, halothane and isoflurane on gap junction conductance in crayfish septate axons: A calcium-and hydrogenindependent phenomenon potentiated by caffeine and theophylline, and inhibited by 4-aminophyridine. J. Membrane Biol., 121, 67-78.

7) Sandra Citi. (1993) The molecular organization of tight junctions. J. Cell Biol., 121, 485-489.

8) Stevenson, B.R. \& Goodenough, D.A. (1984) Zonulae Occludentes in Junctional complex-enriched fractions from mouse liver: Preliminary morphological and biochemical characterization. J. Cell Biol., 98, 1209-1221. 